\title{
Weiße Wasserspeicher - Analyse und Modellierung der Schneedichte in den österreichischen Alpen und Alpenvorländern
}

\author{
Stefan Achleitner · Johannes Schöber
}

Online publiziert: 13. März 2017

(C) Der/die Autor(en) 2017. Dieser Artikel ist eine Open-Access-Publikation.

Zusammenfassung Im diesem Beitrag wird ein empirisches, statistisch basiertes Schätzmodell der Schneedichte basierend auf einem österreichweiten Datensatz von knapp 50.000 Messungen der Schneehöhe und des Schneewasserwertes präsentiert. Das bewusst einfach gehaltene Modell verzichtet auf eine physikalische oder konzeptionell basierte Schneedeckenmodellierung und erlaubt die Umrechnung von gemessen Schneehöhen in Schneewasserwerte.

Aufgrund der geringeren zeitlichen und räumlichen Variabilität der Schneedichte wird diese in Abhängigkeit von der Schneehöhe modelliert, wobei der übliche saisonale Verlauf von Schneehöhe und Dichte berücksichtigt wird. Ebenso ist die Lage (Region und Höhe) durch spezifische Anpassungen im Modell berücksichtigt. Letztlich wird die modellierte Dichte zusammen mit der gemessenen Schneehöhe verwendet, um den Schneewasserwert zu berechnen. Die Resultate zeigen, dass die gewählte Methodik auch in ganz Österreich zu plausiblen Abschätzungen von Schneewasseräquivalent und Dichte führen.

Die Anwendungen in der wasserwirtschaftlichen Praxis sind dabei vielfältig. Neben der schnellen Abschätzung des Schneewasserwerts im Feld oder der Umrechnung von Einzelmessungen, ist auch die räumliche An-

Assoz.-Prof. DI Dr. S. Achleitner ( $\bowtie)$ Arbeitsbereich Wasserbau, Institut für Infrastruktur, Universität Innsbruck, Technikerstraße 13, 6020 Innsbruck, Österreich

stefan.achleitner@uibk.ac.at

Mag. Dr. J. Schöber

Abteilung Wasserkraftplanung,

TIWAG - Tiroler Wasserkraft AG,

Eduard-Wallnöfer-Platz 2, 6020 Innsbruck,

Österreich

johannes.schoeber@tiwag.at wendung gegeben. Aktuell und auch in der Vergangenheit existieren wesentlich weniger Standorte, an denen regelmäßig Schneewasserwert gemessen wird, als Standorte, an denen die Schneehöhe regelmäßig gemessen wird. Das Modell ermöglicht somit Aussagen zum Schneewasserwert mit einer höheren räumlichen Genauigkeit als es nur infolge der seltenen Wasserwertmessungen alleine möglich wäre. Eine weitere Anwendungsmöglichkeit ist die Ermittlung von Klimatologien des Schneewasserwertes auf Basis historischer Schneehöhenmessungen. Auf Basis aktueller Schneehöhenmessungen aus dem Stationsnetz sind verstärkt Anwendungen zur Erfassung des aktuell als Schnee gespeicherten Wassers im Fokus. Dies ist insbesondere in Einzugsgebieten von Interesse, deren Abfluss zur Wasserkrafterzeugung genutzt wird.

White water reserves - analysis and modeling of snow density in the Austrian Alps and the Alpine Foreland

Abstract This article presents an empirical, statistical snow-density and snow-water-equivalent model based on an Austria-wide dataset of ca. 50,000 snow-depth measurements. The intentionally simple model dispenses with physical or conceptual snowpack modeling and makes it possible to calculate the snow water equivalent on the basis of snow depth.

Due its the low temporal and spatial variability, snow density is modeled as a function of snow depth, taking into account the usual seasonal changes in snow depth and density. Similarly, by making individual adjustments to the model, the location (region and altitude) is also taken into consideration. The modeled density, together with the measured snow depth, are then used to calculate the snow water equivalent. The results show that the chosen methodology can be used to provide valid estimations of the snow water equivalent and density throughout Austria.

There are various applications for this approach in practical water resource management: rapid field estimates of the snow water equivalent, conversion of individual measurements, and spatial applications. Today, as in the past, there are significantly fewer locations where the snow water equivalent is regularly measured than there are locations where the snow depth is regularly measured. This model allows more spatially accurate statements regarding the snow water equivalent to be made than would be possible on the basis of infrequent water equivalent measurements alone. A further application is climatological determination of the snow water equivalent on the basis of historical snowdepth measurements. There is also an increasing focus on applications to determine the amount of water currently stored as snow using the latest snowdepth measurements from the network of stations. This is of particular interest in drainage areas where the outflow is used to generate hydropower.

\section{Einleitung}

Im alpinen Raum ist der Schnee von außerordentlicher Bedeutung, da in alpinen Lagen rund 30 bis $50 \%$ der Jahresniederschlagsmenge in Form von Schnee fallen. Die quantitative Erfassung der Schneelage basiert in Österreich auf einem umfassenden Messnetz, was der alpinen Topografie des Landes geschuldet ist. Das Messen der Schneemengen ist dabei für verschiedenste Anwendungsbereiche interessant. Der Wintertourismus ist entsprechend sensibel hinsichtlich der Schneelage, wobei ein immer weiter steigender Aufwand in der Beschneiung von Skigebieten gegeben 


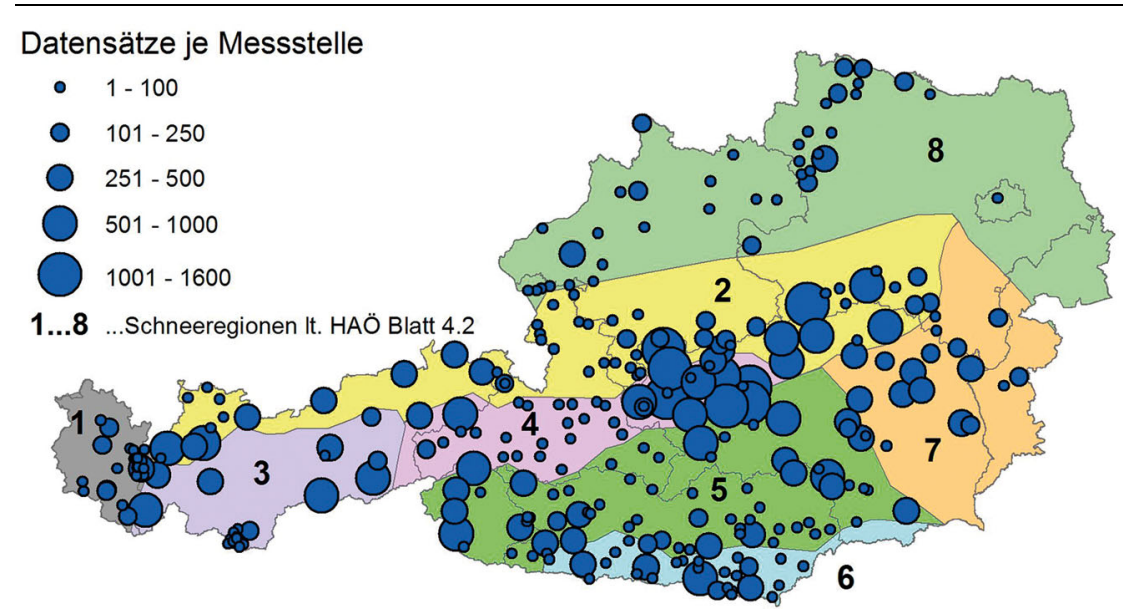

Abb. 1 Schneewasserwertmessungen in Österreich zwischen 1949 und 2010. Punkte stellen die Messorte und die jeweilige Datensatzgröße dar

\section{Messstellen \\ - Schneewasserwertmessung \\ - Schneehöhenmessungen}

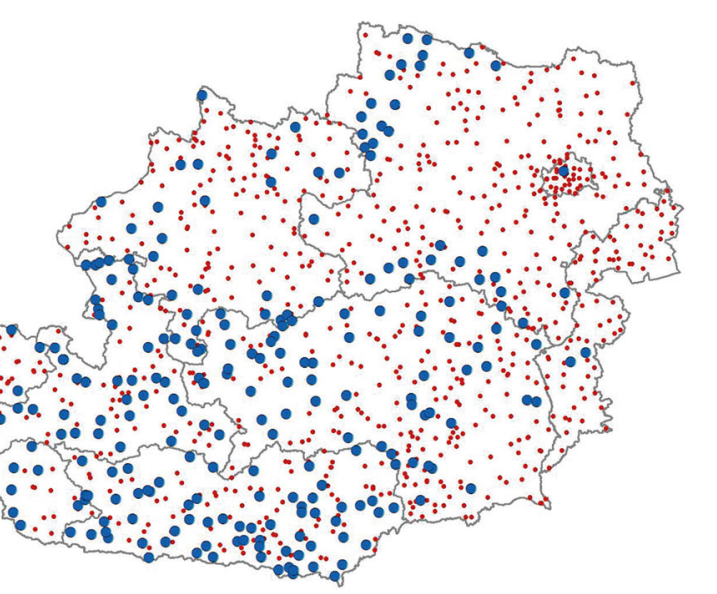

Abb. 2 Räumliche Abdeckung Österreichs durch Schneehöhen- und Schneewasserwertmessungen ist (Steiger 2010; Abegg und Steiger 2016). Aus baulicher Sicht ist die Erfassung von Schnee bzw. Schneelasten ein wichtiges Element zur Bemessung von Bauwerken oder zur Einschätzung der Lawinengefährdung (Fromm 2012, Rhyner et al. 2010). Auch im Bereich der Abflussentstehung spielen Schneeschmelzprozesse eine wichtige Rolle. Hochwasser und Überflutungen in den Alpen und den Voralpen stehen häufig im Zusammenhang mit Schneeschmelzereignissen oder Regen-auf-SchneeEreignissen (Rössler et al. 2014; Merz und Blöschl 2003). Die Modellierung von Schneeprozessen in Abflussprognosemodellen ist daher bereits eine fixer Bestandteil in vielen Real-timePrognosemodellen. Schneemessungen stellen dabei, neben dem Abfluss, eine wichtige Zielgröße zur Kalibrierung der Modelle dar (Achleitner et al. 2012; Schöber et al. 2010; Nester et al. 2012). Ebenso spielt der Schnee bei wasserwirtschaftlichen Betrachtungen eine wichtige Rolle. Die Erfassung des im Einzugsgebiet über die Wintermonate natürlich gespeicherten Wassers ist eine wichtige Eingangsgröße für die Betriebsplanung in der Wasserkraft- und Energiewirtschaft (Barnett et al. 2005; Skaugen et al. 2012). Das vorrangige Ziel (schnee-)hydrologischer Untersuchungen besteht darin, Schneewasserwerte (snow water equivalent - SWE) zu prognostizieren.

Generell hat die Erfassung der Schneeverhältnisse in Österreich eine lange Tradition. Messungen des Schnee-
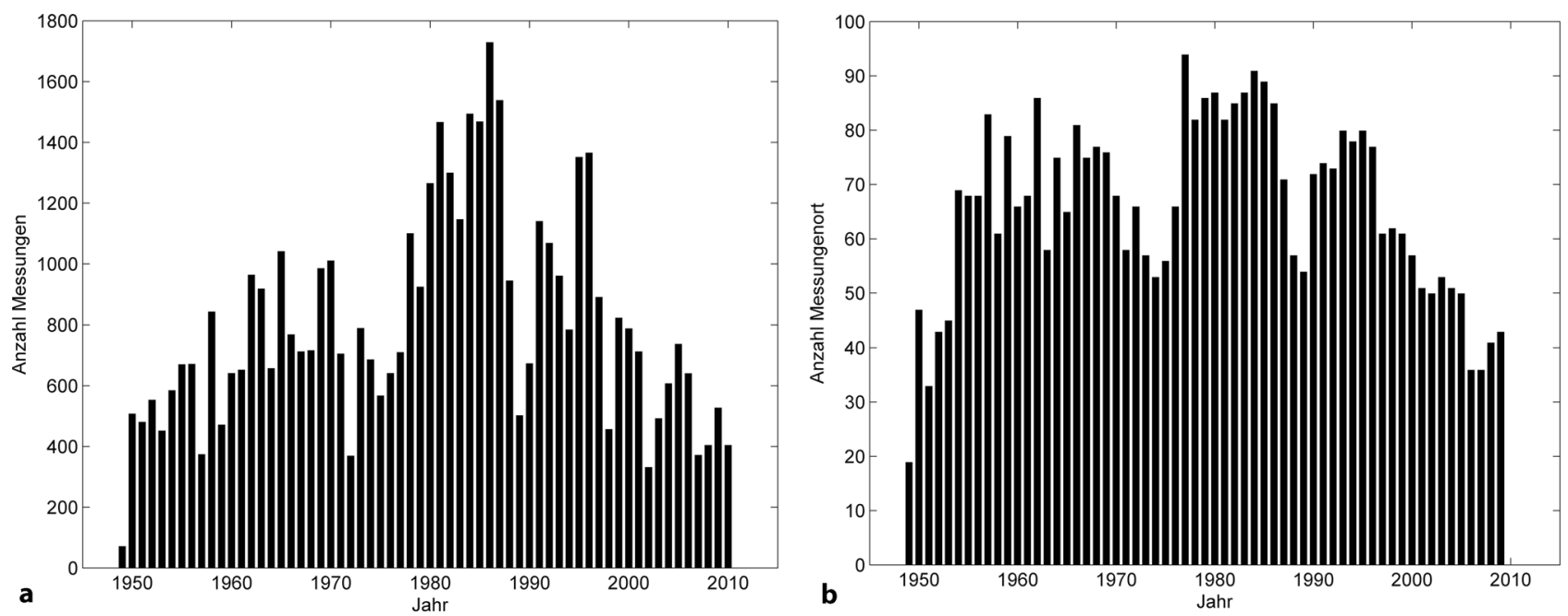

Abb. 3 Zeitlicher Verlauf der Anzahl der (a)Stationen und (b) Anzahl der Messdatensätze an SWE und Schneedichtemessungen, die in den Hydrographischen Jahrbüchern Österreichs veröffentlicht wurden 

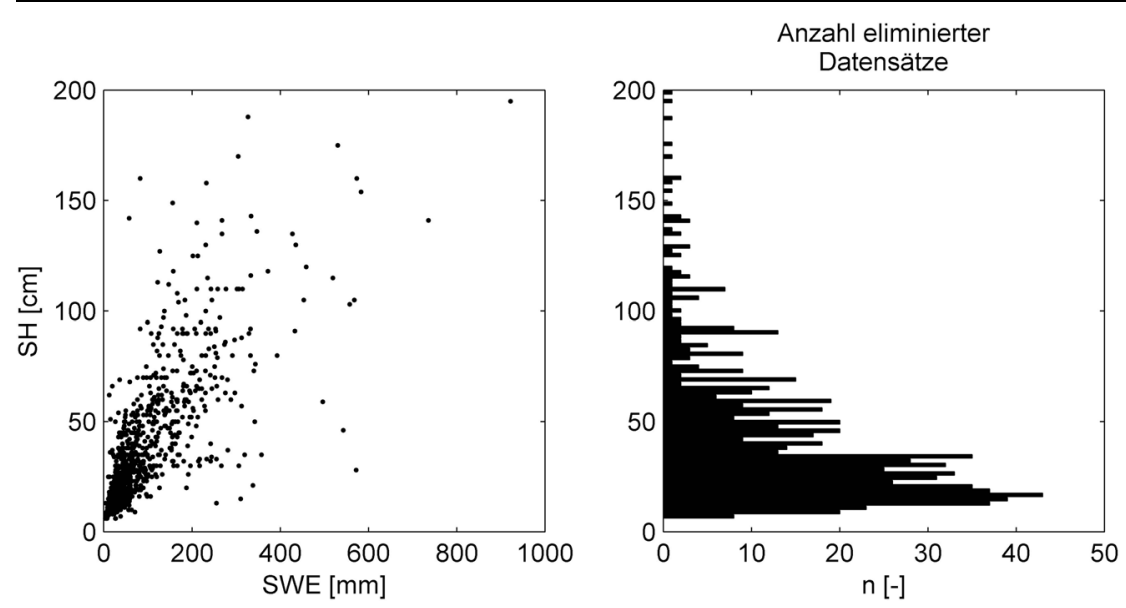

Abb. 4 Verteilung der aus dem Gesamtdatensatz eliminierten Daten
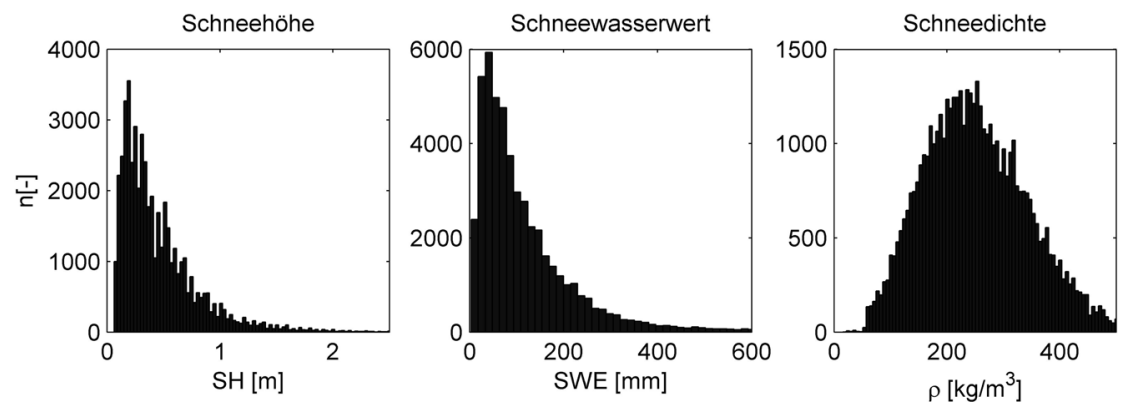

Abb. 5 Häufigkeitsverteilung derSchneehöhe(SH), Schneewasserwert(SWE) und der Dichte $(\rho)$

wasserwerts sind jedoch in Österreich und auch weltweit seltener verfügbar als z. B. die Schneehöhe (SH) (Sturm et al. 2009; Schöber et al. 2016). Große Schneehöhen enthalten nicht zwangsläufig große Wassermengen - entscheidend dafür ist die Schneedichte. Ebenso sind für Schneehöhen weitgehend kontinuierliche Messreihen vorhanden, was bei Schneewasserwerten nicht oder nur vereinzelt der Fall ist. Dies liegt zum Teil an den Messmethoden, da kontinuierliche Messverfahren wie Schneekissen, Waagensysteme oder Schneebänder nicht flächendeckend installiert sind, bzw. erst in der jüngeren Vergangenheit entwickelt wurden. Die in Österreich längste durchgehend gemessene Reihe von SWE ist an der Messstation des Lysimeters Kühtai (Kirnbauer und Blöschl 1990) gegeben. Eine weiter zurückreichende Methode der Schneemessung ist das händische Messen der Schneedichtemessungen, wobei hier aufgrund des nötigen Aufwands größere Zeitabstände zwischen den Messungen liegen.

Um den Aufwand bei Schneedichtemessungen $\mathrm{zu}$ reduzieren, wurde vielfach daran gearbeitet, Methoden zu dichte mittels eines empirischen Modellansatzes. Das Ziel ist die Abschätzung der Schneedichte ( $\rho$ ) mittels der gemessenen Schneehöhe (SH) und grundsätzlichen Standorteigenschaften, wodurch diese sich aufgrund der einfachen Anwendbarkeit auch in der Praxis und im Feld eignen. Derartige empirische Modelle (z. B. Rohrer und Braun 1994; Jonas et al. 2009; Sturm et al. 2010; López-Moreno et al. 2013) sind entsprechend einfacher in der Anwendung, da weniger Daten benötigt werden, haben aber dennoch eine entsprechende Belastbarkeit.

Eine mögliche Anwendung dabei ist es, kontinuierliche und mit erheblich größerer Flächenabdeckung gemessene reine Schneehöhendaten in Schneewasserwerte überführen. Derartige lineare Modelle, die den Zusammenhang zwischen Schneehöhe und Dichte herstellen, wurden in der letzten Zeit auch auf Einzugsgebiets- bzw. Regionalskala angewendet. Grundsätzlich wird der Vorteil genutzt, dass die räumliche und zeitliche Variabilität der Schneedichte geringer ist als die der Schneehöhe oder des Schneewasserwerts (Elder et al. 1998; Mizukami und Perica 2008). Jörg-Hess et al. (2014) verwendeten ein solches Modell zur Ableitung einer Klimatologie des Schneewasserwertes für die Schweiz. Schöber et al. (2014) wendeten die Methode in hochalpinen Einzugsgebieten (vergletscherte Gebiete zwischen 2.000 und $3.400 \mathrm{~m}$. ü. A.) an, um Schneehöhen aus Laserscanbefliegungen in Schneewasserwerte überzuführen.

Im Alpenraum wurden solche Modelle von Jonas et al. (2009) für die Schweiz und von Schöber et al. (2016) für Tirol vorgestellt. Die hier vorgestellte Arbeit stellt eine räumliche Erweiterung des Modells aus Schöber et al. (2016) dar und umfasst Datengrundlagen für Österreich.

\section{Daten}

In der vorliegenden Arbeit wird mit einem gesamtösterreichischen Datensatz gearbeitet, der Messwerte aus den hydrografischen Jahrbüchern seit 1949 umfasst. Die hydrografischen Dienste von Österreich haben seit Jahrzehnten umfassende Messungen der Schneedichte und des Schneewasseräquivalents durchgeführt. Dies erfolgte überwiegend manuell durch Beobachter und das Anlegen von Schneeschächten und Abstechen von Schneeproben. 


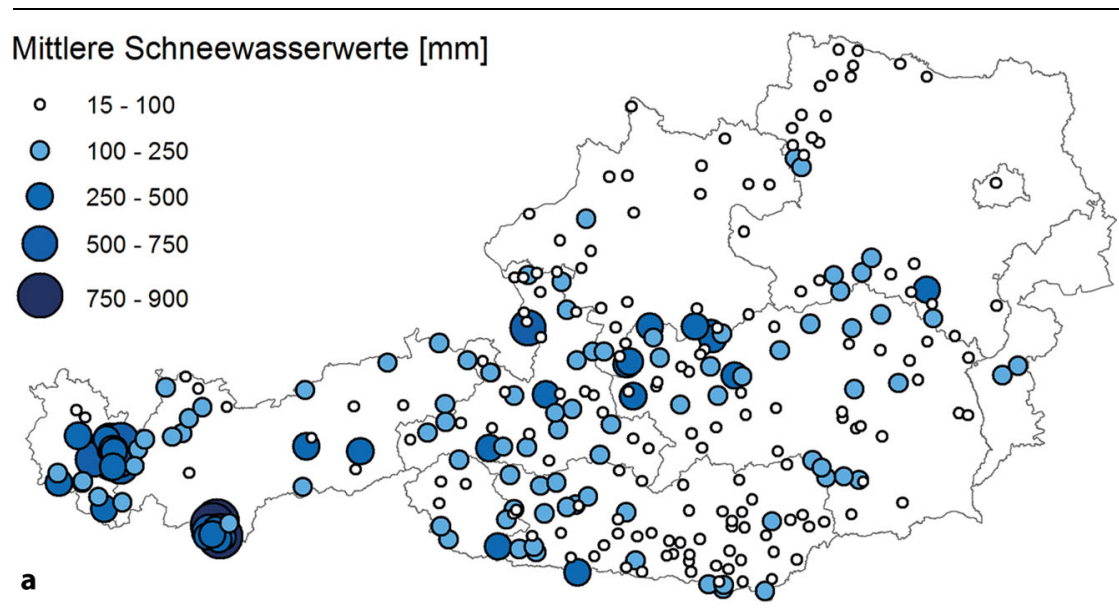

Mittlere Schneedichte $[\mathrm{kg} / \mathrm{m} 3]$
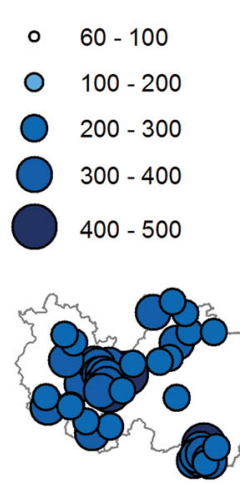

b

Abb. 6 Räumliche Verteilung der mittleren (a) Schneewasserwerte und (b) Schneedichten je Station

Die Erfassung der Schneemenge erfolgte mittels Wiegung vor Ort oder früher vereinzelt auch durch im Labor geschmolzene Proben. Daraus lässt sich die Masse der Schneeprobe messen sowie Dichte und Schneewasseräquivalent bei Kenntnis der Schneehöhe berechnen. Diese Messungen werden seit den 1950er-Jahren regelmäßig in den „Hydrographischen Jahrbüchern“ der Schneewasserwerte ist in Abb. 2 im Vergleich zu Standorten dargestellt, an welchen nur Schneehöhe gemessen wird. Es ist leicht ersichtlich, dass Schneehöhendaten eine bei weitem größere Abdeckung haben als Schneewasserwerte. Um auch die Schneehöhenmessungen nutzbar zu machen, wurden gerade in den letzten Jahren statistische Schneedichtemodelle entwickelt (Jonas et al. 2009; Sturm et al. 2010; Schöber et al. 2016).

In Abb. 3 ist die Verfügbarkeit von Messstationen und Datensätzen über die Zeit abgebildet. Daraus ist ersichtlich, dass die Erfassung von Schneedaten in den 1980er- und 1990er-Jahren sehr intensiv betrieben wurde. In den 2000er-Jahren sank die Anzahl der erfassten Stationen und Datensätze auf das Niveau der in den 1950er-Jahren betriebenen Schneemessungen. In vielen Flussgebieten (teils auch alpinen Flussgebieten) werden derzeit keine SWE-Messungen mehr durchgeführt.

Da ein Großteil der Daten nicht digital vorliegt, wurden die Jahrbücher gescannt und eine automatische Texterkennung angewandt. Es erfolgte eine Prüfung der Daten nach Plausibilität. Neben dem Test, ob die erfassten Dichten, Schneewasserwerte und Höhen stimmig sind, erfolgte eine Einschränkung der Datenbandbreite. So wurden einerseits Datensätze ausgeschieden, deren aus Schneehöhe und Wasserwert gerechnete Dichte von der aus dem Jahrbuch erfassten Dichte stark abwichen. Weiters wurden nur Datensätze mit Dichten in der Bandbreite von $\rho=50-600 \mathrm{~kg} / \mathrm{m}^{3}$ berücksichtigt. Bei Schneedecken mit Dichten kleiner $50 \mathrm{~kg} / \mathrm{m}^{3}$ würde es sich ausschließlich um Neuschnee handeln - entsprechende Daten wurden ausgeschlossen. Sehr große Dichten hingegen kommen nur selten vor. Hinsichtlich der Schneehöhe

Tab. 1 Schneemessdatensätze der hydrografischen Dienste. Verfügbare Datensätze nach Datenprüfung in verschiedenen Schneeregionen und Höhenzonen

\begin{tabular}{|l|l|l|l|l|l|l|l|l|l|l|}
\hline & Region & 1 & 2 & 3 & 4 & 5 & 6 & 7 & 8 & Gesamt \\
$\begin{array}{l}\text { Höhen- } \\
\text { zone }\end{array}$ & $\leq 1400 \mathrm{~m}$ & 809 & 13655 & 2287 & 8223 & 12288 & 2393 & 3024 & 2320 & 44999 \\
\hline & $(1950-2008)$ & $(1949-2010)$ & $(1952-2010)$ & $(1949-2010)$ & $(1949-2010)$ & $(1949-2001)$ & $(1949-2010)$ & $(1949-2010)$ & \\
& $>1400 \mathrm{~m}$ & 903 & 523 & 1450 & 900 & 265 & 280 & 405 & 0 & 4726 \\
\hline & $(1950-2006)$ & $(1949-2010)$ & $(1952-2010)$ & $(1949-2010)$ & $(1949-2000)$ & $(1949-2001)$ & $(1966-1985)$ & $(-)$ & \\
\hline & Gesamt & 1712 & 14178 & 3737 & 9123 & 12553 & 2673 & 3429 & 2320 & 49725 \\
\hline
\end{tabular}

Tab. 2 Regionsspezifischer Offset der Regressionsfunktion OSreg $\left[\mathrm{kg} / \mathrm{m}^{3}\right]$

\begin{tabular}{l|l|l|l|l|l|l|l|l|l|l|}
\hline & Region/Datensatz & 1 & 2 & 3 & 4 & 5 & 6 & 7 & 8 \\
Höhen- & $\leq 1400 \mathrm{~m}$ & Österreich & 26.53 & 45.45 & -16.56 & 12.49 & -33.45 & 11.96 & 22.54 & - \\
zone & $>1400 \mathrm{~m}$ & Österreich & 30.45 & 10.03 & 9.83 & -3.24 & -0.72 & -27.95 & -5.18 & -17.29 \\
\hline
\end{tabular}



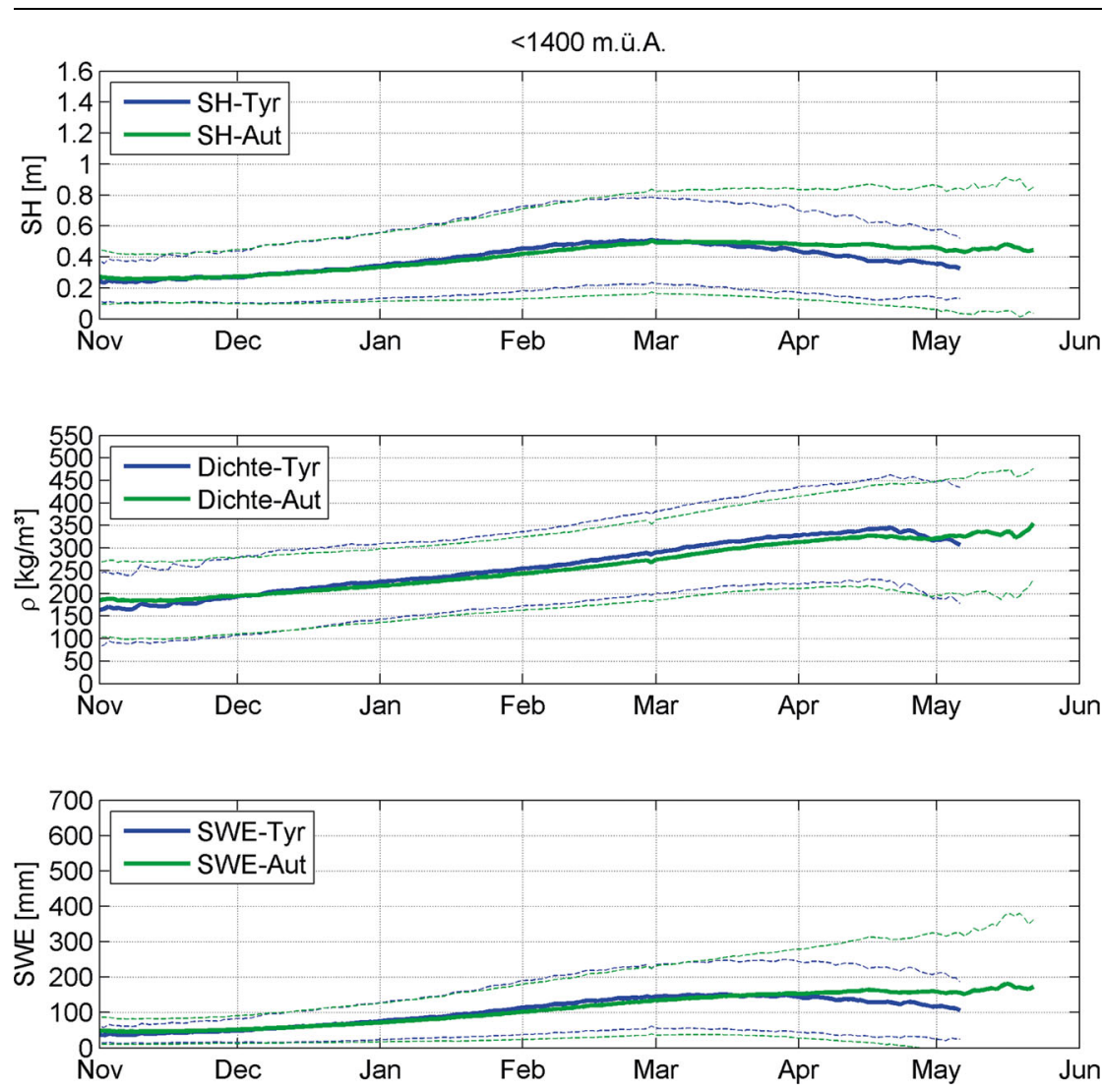

Abb. 7 Jahresverlauf des mittleren Schneewasserwerts (SWE), der mittleren Schneehöhe $(\mathrm{SH})$ und derDichte ( $\rho)$ für Gesamtösterreichund den Ausschnitt Tirol in derHöhenzone $<1.400$ m. ü. A. (strichlierte Linien entsprechen jeweils \pm der Standardabweichung)

erfolgte die Einschränkung auf den Bereich $\mathrm{SH}<5 \mathrm{~cm}$, da bei kleinen Schneehöhen mit großen Messunsicherheiten $\mathrm{zu}$ rechnen wäre. Insgesamt verbleiben nach Datenprüfung und Einschränkung 49.724 Datensätze. Die entfernten Datensätze umfassten primär die kleinen Schneehöhen bzw. Wasserwerte (Abb. 4).

Die Häufigkeitsverteilungen des Gesamtdatensatzes für SH, SWE und Dichte folgen dabei dem typischen Muster (siehe Abb. 5). Die Dichte $\rho$ ist dabei annähernd normal verteilt wobei die Parameter SWE und SH eine log-normal-Verteilung aufweisen (vgl. Jonas et al. 2009; Sturm et al. 2010).

Das statistische Schneedichtemodell nutzt den Umstand, dass die Schneehöhe oder die Schneewasserwerte räumlich und zeitlich zwar stark variieren, die Schneedichte selbst jedoch deutlich weniger Variation aufweist (siehe Abb. 6).

\section{Methode}

Das grundlegende Ziel ist es, die aktuelle Schneedichte auf Basis der gemessen Schneehöhe, des Zeitpunkts sowie der Information über die Lage des Standorts zu prognostizieren. Dabei hat das Dichtemodell folgende Form:

$$
\rho_{s}=f\left(S H_{m},\right. \text { Tag im Jahr, }
$$$$
\text { Region und Höhenlage) }
$$

Die ermittelte Dichte wird mit der Schneehöhe in Schneewasserwerte umgerechnet.

$$
S W E_{\text {sim }}=S H_{\text {obs }} \cdot \rho_{\text {sim }}
$$

Die Methodik beruht im Wesentlichen auf der vereinfachten Annahme, dass die Schneedichte als eine Funktion der Schneehöhe beschrieben werden kann (Jonas et al. 2009; Sturm et al. 2010). Unter konstanten meteorologischen Bedingungen wäre die Schneedichte tatsächlich wesentlich durch die Schneeakkumulation über die Zeit geprägt. Da dies in den Alpen nicht der Fall ist, werden die Daten vor der Berechnung der Regressionsparameter gruppiert. Es ist kaum verwunderlich, dass sich in früheren Untersuchungen die Seehöhe der Messstation als relevanter Parameter erwiesen hat. In den unterschiedlichen Höhenzonen ist mit geänderten Temperatur- und Niederschlagsverhältnissen $\mathrm{zu}$ rechnen, weshalb sich auch die Schneehöhe unterscheidet. Dabei hat sich in alpinen Verhältnissen die Höhe von 1.400 m. ü. A. als geeignete Grenze zur Trennung von Datensätzen erwiesen (Jonas et al. 2009; Schöber et al. 2016). Ebenfalls ist es notwendig, die Daten nach der Zeit/ Saison zu gruppieren, da die Schneedichte im Fall von Neuschnee gering ist (etwa $100 \mathrm{~kg} / \mathrm{m}^{3}$ ), aber im Frühling, wenn während der Schneeschmelze der Wassergehalt in der Schneedecke stark ansteigt, sind Schneedichten zwischen 400 und $500 \mathrm{~kg} / \mathrm{m}^{3}$ möglich. Deshalb liegen die Parameter der zuletzt publizierten statistischen Schneehöhe/ Dichte-Modelle als Funktionen der Zeit in Monatsauflösung (Jonas et al. 2009) oder in Tagesauflösung (Schöber et al. 2016) vor, die den üblichen Verlauf der Wintersaison widerspiegeln sollen.

Als erster Schritt für die Berechnung der Schneeverläufe und der linearen Regressionsparameter wurden demnach die Daten entsprechend der Höhe der Messstationen den zwei Höhenzonen $<1.400 \mathrm{~m}$. ü. A. und $\geq 1.400 \mathrm{~m}$. ü. A. zugeordnet. Um die zeitliche Variation über die Wintersaison hinweg zu berücksichtigen, ist das Ziel, für jeden Tag Modellparameter zu ermitteln. Die Datensätze selbst enthalten keine durchgehenden Reihen in Tagesauflösungen. Meist werden Schneewasserwerte in Wochenintervallen gemessen. Aus diesem Grund werden Messungen im zeitlichen Nahbereich $( \pm 16$ Tage) zusammengefasst (Schöber et al. 2016). Mit den Daten innerhalb dieses gleitenden Zeitfensters erfolgt die lineare Regression zwischen der beobachteten Schneehöhe $\mathrm{SH}_{\mathrm{obs}}$ in [m] und der Schneedichte Dichte $\varrho_{\text {sim }}\left[\mathrm{kg} / \mathrm{m}^{3}\right]$. Schneedaten (SH, Dichte, SWE), die beispielsweise dem 1. Jänner zugeordnet werden, umfassen den Zeitraum Mitte Dezember bis Mitte Jänner aus allen Beobachtungsjahren. Der lineare Zusammenhang ist damit für jeden Tag in der Wintersaison geben durch:

$$
\rho_{\text {sim }}=k \cdot S H_{\text {obs }}+d \text {. }
$$



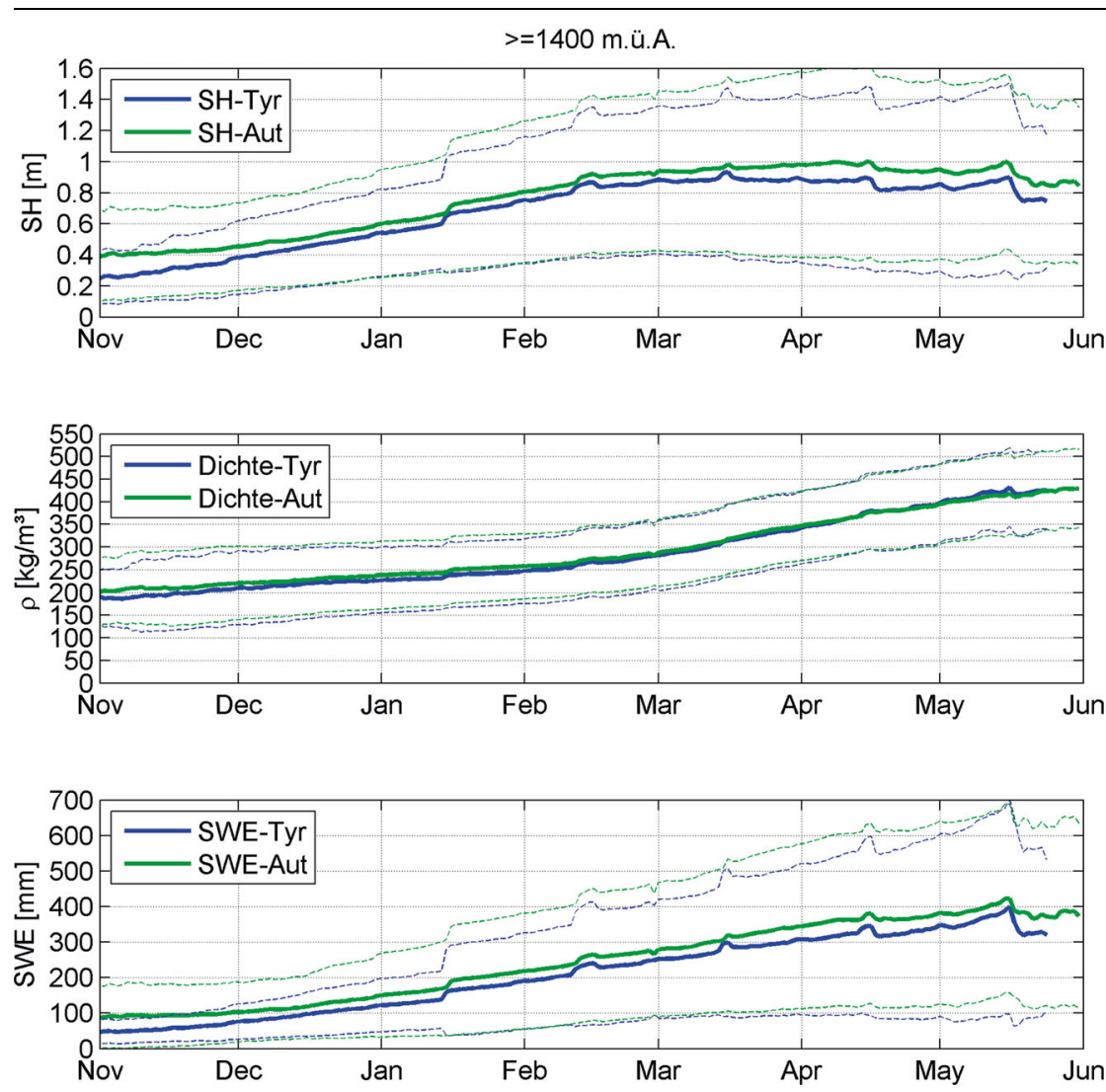

Abb. 8 Jahresverlauf des mittleren Schneewasserwerts (SWE), der mittleren Schneehöhe $(\mathrm{SH})$ und der Dichte $(\rho)$ für gesamt Österreich und den AusschnittTirol in der Höhenzone $\geq 1400$ m. ü. A. (strichlierte Linien entsprechen jeweils \pm der Standardabweichung)

Die tagesspezifischen Parameter ( $\left[\mathrm{kg} / \mathrm{m}^{4}\right]$ und $\mathrm{d}\left[\mathrm{kg} / \mathrm{m}^{3}\right]$ ) der Regressionsgleichung sind österreichweit gültig und nur getrennt nach den Höhenzonen $(<1.400 \mathrm{~m}$. ü. A. und $\geq 1.400$ m. ü. A.) in zwei Klassen unterschiedlich ermittelt.

Zur weiteren räumlichen Anpassung des Modells werden Schneeklimazonen des Hydrologischen Atlas von Österreich herangezogen (Schöner und Mohnl 2003; HAÖ, Kapitel 4.2). Diese acht Schneeregionen in Österreich (Abb. 1) repräsentieren räumliche $\mathrm{Ge}$ gebenheiten wie Höhe und Niederschlagscharakteristik (u. a. Nordstaulage, Zentralalpine Zonen, südalpine Zonen, Vorland). Zur Modellverfeinerung innerhalb dieser Regionen werden die Messungen aus den hydrografischen Jahrbüchern diesen Schneeregionen zugeordnet (Tab. 1). Die nur höhenabhängigen Regressionen (Grenzwert bei $1.400 \mathrm{~m}$. ü. A.) werden angewendet und innerhalb dieser Schneeregionen wird aus dem resultierenden Modellfehler ein regionsspezifisches Residuum $O S_{\text {reg }}$

\section{Ergebnisse und Diskussion}

\subsection{Datenanalyse}

In Abb. 7 und Abb. 8 sind die mittleren Schneehöhen $(\mathrm{SH})$, Schneedichte ( $\rho)$ und Schneewasserwerte (SWE) im Verlauf der Wintersaison jeweils für die Höhenzone über bzw. unter 1.400 m. ü. A. dargestellt. Darin enthalten sind die in Schöber et al. (2016) für Tirol ermittelten Verläufe (blau) und die auf Basis der österreichweiten Daten ermittelten Verläufe (grün). Die strichlierten Linien entsprechen jeweils der positiven bzw. negativen Standardabweichung. In den jahreszeitlichen Verläufen ist grundsätzlich wenig Veränderung durch die Erweiterung des Datenpools von Tirol auf Österreich zu verzeichnen. Einzig gegen Ende der Saison sind leicht höhere Werte in allen Parametern beim österreichischen Datensatz zu erkennen. Dies spricht für die weitere Verwendung der bisher angewendeten Trennung der Datensätze bei $1.400 \mathrm{~m}$. ü. A. Mit einer mittleren Reihenlänge von 33 Jahren sind die Ergebnisse der mittleren Dichte und Schneewasserwerte als klimatologische Mittel zu sehen.

\subsection{Modellergebnisse}

Die Variation der Modellparameter im Jahresverlauf für beide Höhenzonen ist in Abb. 9 dargestellt. Es werden jeweils k (Diagramm oben) und d (Diagramm Mitte) für die Höhenzone $\geq 1400$ m. ü. A. (schwarze Kurven) und für die Höhenzone < 1400 m. ü. A. (rote Kurven) dargestellt. Strichlierte Linien zeigen die 95\%-Konfidenzintervalle der Regressionsparameter. Der Umfang an zur Verfügung stehenden Datensätzen in dem jeweils - zu einem Zeitpunkt - berücksichtigten Zeitfenster (n) ist ebenfalls für beide Höhenzonen dargestellt (Diagramm unten). Entsprechend der schlechteren Datenlage zu Saisonbeginn und Saisonende erhöht sich auch die Breite des 95\%-Konfidenzintervalls. Ebenso ist durch die generell ungünstigere Datenlage in der oberen Höhenzone das 95\%-Konfidenzintervall geringfügig breiter gefasst.

Im Unterschied zum Modell von Jonas et al. (2009) sind die Gradienten k in der Höhenregion unter $1.400 \mathrm{~m}$. ü. A. bis in den März negativ. Dieser Unterschied kann sich durch den Einfluss von Neuschnee ergeben und ist somit auch von den Messintervallen abhän- 

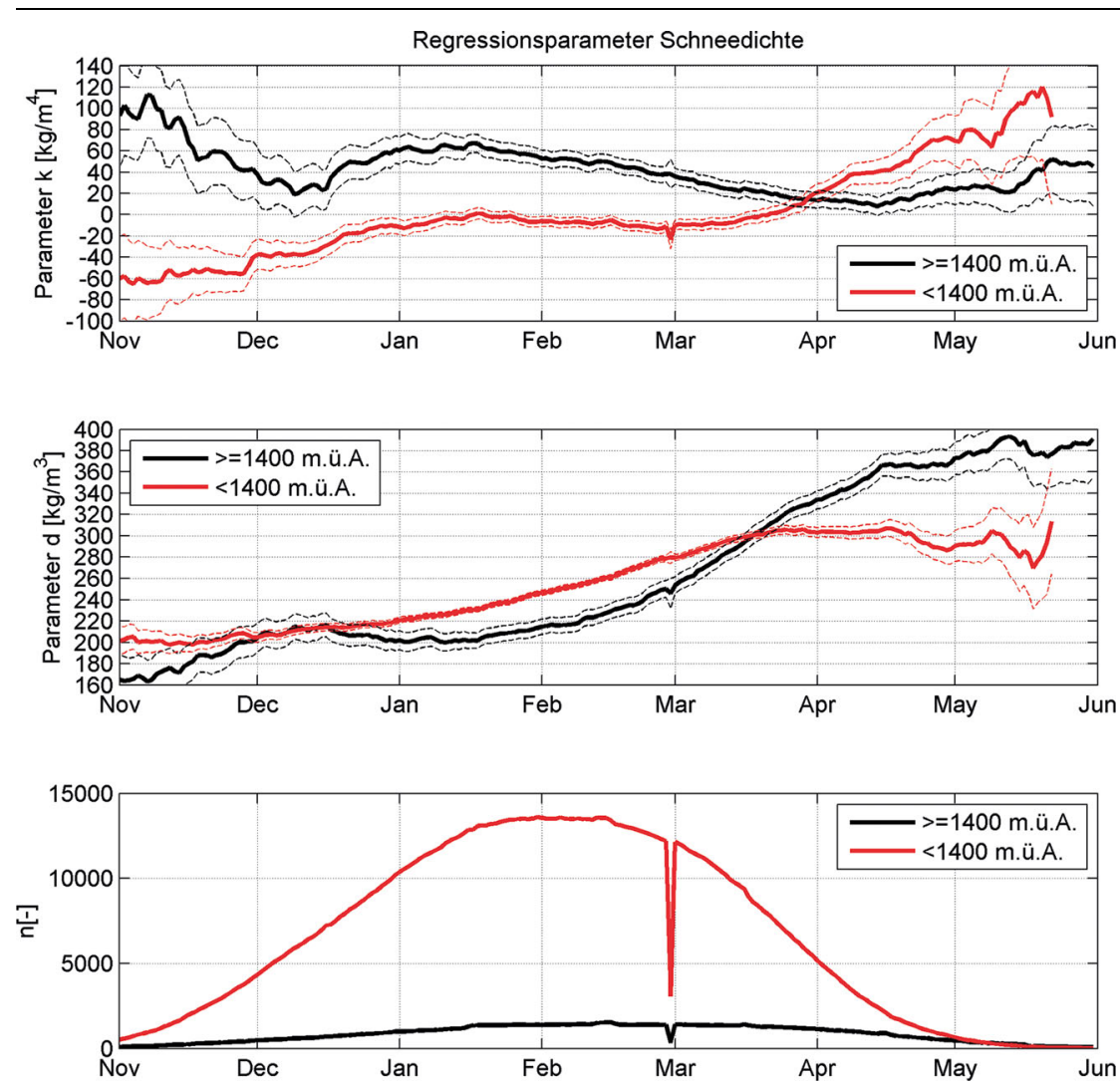

Abb. 9 Jahresverlauf der Modellparameter k und d für die Regression am Gesamtdatensatz ohne regionsspezifischen Offset (strichlierte Linien entsprechen dem 95\%Konfidenzintervall) gig. Österreichische und Schweizer Daten unterscheiden sich dahingehend, dass in Österreich meist wöchentliche Messintervalle, in der Schweiz aber meist zweiwöchige Messintervalle vorliegen. In einer Zeitskale von wenigen Tagen sind $\mathrm{SH}$ und $\rho$ zumeist negativ korreliert, da Neuschnee die Schneedichte reduziert aber gleichzeitig die Schneehöhe ansteigen lässt. In Schöber et al. (2016) wird eine Erweiterung des hier beschriebenen statistischen Modellansatzes präsentiert. Da der Zusammenhang von Neuschneehöhe und Abnahme der Schneedichte nur auf Basis der Daten eines Schneekissens in Tirol ermittelt wurde, wird von einer Anwendung dieser Modellerweiterung in der vorliegenden österreichischen Anwendung abgesehen.

Die ermittelten regionsspezifischen Anpassungen mittels konstanten Offsets $\left(\mathrm{OS}_{\mathrm{reg}}\right)$ sind in Tab. 2 zusammengestellt. Diese Parameter werden zur Berechnung der Schneedichte gemäß Gleichung 5 entsprechend der jeweiligen Schneeregion eingesetzt, aus der die Daten stammen. Abb. 10 und Abb. 11 zeigen die Ergebnisse der Anwendung des Modells auf die Datensätze in den beiden Höhenregionen. Die jeweils linken Diagramme zeigen, dass die Modellfehler der Dichtefunktion eine größere Bandbreite (im Mittel bei ca. $50 \mathrm{~kg} / \mathrm{m}^{3}$ ) aufweisen. Der daraus in Kombination mit der Schneehöhe ermittelte Schneewasserwert führt $\mathrm{zu}$ einer reduzierten Fehlergröße von im


Abb. 10 Modellanwendung und Fehlerbandbreite für die Höhenregion $<1400$ m. ü. A 

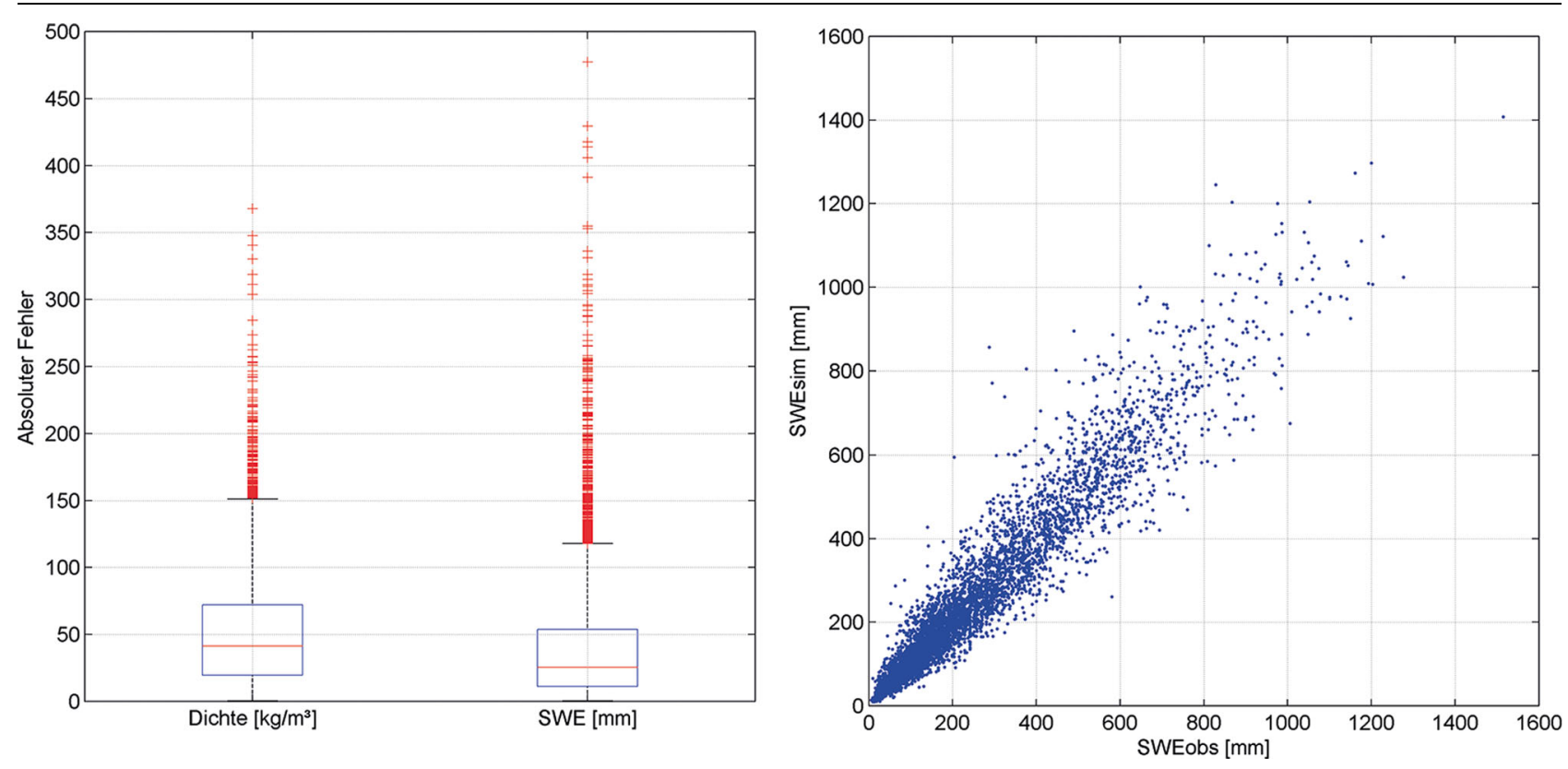

Abb. 11 Modellanwendung und Fehlerbandbreite für die Höhenregion> 1400 m. ü. A

Mittel ca. $20 \mathrm{~mm}$ in beiden Höhenzonen.

In den jeweils rechten Abbildungen sind simulierte und gemessene Schneewasserwerte gegeneinander aufgetragen. Diese Streuung nimmt mit steigendem Schneewasserwert nur leicht zu Das bedeutet, dass relative Fehler bei größeren Schneewasserwerten geringer sind. Weiters zeigte sich, dass relative Fehler im Verlauf der Saison abnehmen. Bezogen auf einzelne Stationen in Tirol ergaben sich relative Modellfehler in der Größenordnung von $13 \%$ bis $25 \%$. Für die Anwendung eines eigenen Schneedichtemodells für die Schneedecke auf Gletschern im April oder Mai ergaben sich noch geringere relative Fehler in der Größenordnung von $5 \%$ bis $2 \%$ (Schöber et al. 2016).

\section{Zusammenfassung}

Auf Basis der Schneemessungen der hydrografischen Dienste von Österreich konnten die mittleren Verläufe von Schneedichte und Schneewasserwert in den vergangenen 60 Jahren ermittelt werden. Es konnten typische saisonale Verläufe ermittelt werden. Dabei folgt die Schneedichte einem zumeist typischen saisonalen Verlauf, Schneehöhe und auch Schneewasserwert hingegen können von Jahr zu Jahr - in Abhän- gigkeit von der Niederschlagsmenge stark variieren.

Das vorgestellte Modell zur Ermittlung der Schneedichte und des Schneewasserwerts auf Basis einer Schneehöhenmessung zeigt für den verwendeten österreichischen Datensatz eine gute Anpassung. Die Anwendung berücksichtigt zeitliche Variationen im Verlauf der Saison und erlaubt eine durchgehende, tagesweise Anwendung. Zudem sind regions- und höhenabhängige Unterschiede und Variationen berücksichtigt. Um die Schneedichte an einem bestimmten Tag im Winter (zwischen November und Mai) berechnen zu können, stehen die Regressionsparameter $\mathrm{k}$ und $\mathrm{d}$ in Tagesauflösung zur Verfügung. Die Parameter k und d können der Abb. 9 - entsprechend der Höhenlage der Messung - entnommen werden. Alternativ besteht die Möglichkeit, die taggenauen Regressionsparameter $\mathrm{k}$ und $\mathrm{d}$ bei den Autoren anzufragen. Zusammen mit dem Messwert der Schneehöhe und dem regionsspezifischen Offset (Tab. 2) werden diese in die Gleichung 5 eingesetzt. Somit kann die Schneedichte rasch geschätzt und der Schneewasserwert berechnet werden.

Mögliche Anwendungen des Modells reichen von einer Verdichtung historischer Datenreihen mittels gemessener
Schneehöhen an Stationen bis zur tagesaktuellen Abschätzung von Schneewasserwerten. Das zur Verfügung stehende Schneedichtemodell ermöglicht die Umrechnung von Schneehöhendaten, unabhängig davon, ob diese als Einzelmessungen oder flächige Daten aus z. B. Laserscanbefliegungen vorliegen. Für die Wasserwirtschaft ist dabei die Ermittlung von gespeicherten Wassermengen in Einzugsgebieten für eine mögliche Optimierung der Kraftwerksbetriebsführung von Bedeutung.

Open access funding provided by University of Innsbruck and Medical University of Innsbruck.

Open Access Dieser Artikel wird unter der Creative Commons Namensnennung 4.0 International Lizenz (http:// creativecommons.org/licenses/by/4. $0 /$ deed.de) veröffentlicht, welche die Nutzung, Vervielfältigung, Bearbeitung, Verbreitung und Wiedergabe in jeglichem Medium und Format erlaubt, sofern Sie den/die ursprünglichen $\mathrm{Au}$ tor(en) und die Quelle ordnungsgemäß nennen, einen Link zur Creative Commons Lizenz beifügen und angeben, ob Änderungen vorgenommen wurden. 
Abegg, B., Steiger, R. (2016): Herausforderung Klimawandel: Alpiner Skitourismus unter Anpassungsdruck. Geographische Rundschau 2016/5, 16-21.

Achleitner, S., Schöber, J., Rinderer, M., Leonhardt, G., Schöberl, F., Kirnbauer, R., Schönlaub, H. (2012): Analyzing the operational performance of the hydrological models in an alpine flood forecasting system. In: Journal of Hydrology 412-413, 90-100. (10.1016/j.jhydrol.2011.07. 047)

Barnett, T. P., Adam, J. C. und Lettenmaier, D. P. (2005): Potential impacts of a warming climate on water availability in snowdominated regions. Nature 438 (7066), 303-309.

Bartelt, P. und Lehning, M. (2002): A physical SNOWPACK model for the Swiss avalanche warning: Part I: numerical model. Cold Reg. Sci. Technol. 35 (3), 123-145.

Brun, E., Martin, E., Simon, V., Gendre, C. \& Coleou, C. (1989): An energy and mass model of snow cover suitable for operational avalanche forecasting. J. Glaciol. 35 (121), 333-342.

Elder, K., Rosenthal, W. und Davis, R. E. (1998) Estimating the spatial distribution of snow water equivalence in a montane watershed. Hydrol. Process. 12 (10-11), 1793-1808.

Frey, S. und Holzmann, H. (2015): A conceptual, distributed snow redistribution model, Hydrol. Earth Syst. Sci., 19, 4517-4530, doi:10.5194/hess19-4517-2015.

Fromm R. (2012): Automatische Messstationen im Hochgebirge - ein Beitrag zum Schutz vor Naturgefahren. Newsletter des Instituts für Naturgefahren und Waldgrenzregionen des BFW, Innsbruck, (1): $1 \mathrm{~S}$

Jonas, T., Marty, C. \& Magnusson, J. (2009): Estimating the snow water equivalent from snow depth measurements in the Swiss Alps. J. Hydrol. 378 (1-2), 161-167.
Jörg-Hess, S., Fundel, F., Jonas, T. und Zappa, M. (2014): Homogenisation of a gridded snow water equivalent climatology for Alpine terrain: methodology and applications. Cryosphere 8 (2), 471-485.

Kirnbauer, R. and Blöschl, G. (1990): A lysimetric snow pillow station at Kühtai/Tyrol. In Hydrology in Mountainous Regions. I - Hydrological Measurements; the Water Cycle (Proceedings of two Lausanne Symposia, August 1990) IAHS Publ. no. 193, 1990. http://hydrologie.org/ redbooks/a193/iahs_193_0173.pdf

López-Moreno, J. I., Fassnacht, S. R., Heath, J. T., Musselman, K. N., Revuelto, J., Latron, J., Morán-Tejeda, E. \& Jonas, T. (2013): Small scale spatial variability of snow density and depth over complex alpine terrain: Implications for estimating snow water equivalent. Adv. Water Resour. $55,40-52$.

Merz, R. und Blöschl, G. (2003): A process typology of regional floods. Water Resour. Res. 39 (12), 1340-1359.

Mizukami, N. und Perica, S. (2008): Spatiotemporal characteristics of snowpack density in the mountainous regions of the western United States. J. Hydrometeorol. 9 (6), 1416-1426.

Nester, T., Kirnbauer, R., Parajka, J. \& Blöschl, G. (2012): Evaluating the snow component of a flood forecasting model. Hydrol. Res. 43 (6), 762-779.

Rhyner J., Voigt T., Marty C., Dobesberger P., Fromm R., Solheim A., Vojtek M., Gab K. (2010): Avalanches. In: Impacts of climate change on snow, ice, and permafrost in Europe, ETC/ACC Technical Paper (13): 92-97

Rohrer, M. B. und Braun, L. N. (1994): Longterm records of snow cover water equivalent in the Swiss Alps 2. Simulation. Nord. Hydrol. 25 $65-78$
Rössler, O., Froidevaux, P., Börst, U., Rickli, R., Martius, O. und Weingartner, R. (2014): Retrospective analysis of a nonforecasted rain-onsnow flood in the Alps - a matter of model limitations or unpredictable nature? Hydrol. Earth Syst. Sci. 18 (6), 2265-2285

Schöber, J., Achleitner, S., Kirnbauer, R., Schöberl, F. und Schönlaub, H. (2010): Hydrological modelling of glacierized catchments focussing on the validation of simulated snow patterns applications within the flood forecasting system of the Tyrolean river Inn. Adv. Geosci. 27, 99-109. Schöber, J., Schneider, K., Helfricht, K., Schattan, P., Achleitner, S., Schöberl, F. \& Kirnbauer, R. (2014): Snow cover characteristics in a glacierized catchment in the Tyrolean Alps - Improved spatially distributed modelling by usage of Lidar data. J. Hydrol. 519 (2014), 3492-3510.

Schöber, J., Achleitner, S., Bellinger, J., Kirnbauer, R. \& Schöberl, F. (2016): Analysis and modelling of snow bulk density in the Tyrolean Alps. Hydrol.Res. 47.2, 419-441.

Schöner, W. und Mohnl, H. (2003): Schneehöhen und Schneebedeckung (Blatt 4.2), in Hydrologischer Atlas Österreich, Bundesministerium für Land- und Forstwirtschaft, Umwelt und Wasserwirtschaft, Abteilung Wasserhaushalt; (ISBN 3-85437-250-7);

Skaugen, T., Bache Stranden, H. und Saloranta, T. (2012):Trends in snow water equivalent in Norway (1931-2009). Hydrol. Res. 43 (4), 489-499.

Steiger, R. (2010): The impact of climate change on ski season length and snowmaking requirements in Tyrol, Austria. In: CLIMATE RESEARCH $43 / 3$, S. 251-262.

Sturm, M., Taras, B., Liston, G. E., Derksen, C., Jonas, T. \& Lea, J. (2010): Estimating snow water equivalent using snow depth data and climate classes. J. Hydrometeorol. 11 (6), 1380-1394. 